\title{
Eine neue Sozialphilosophie auf Kantischer Basis.
}

Von Felix Krueger in Kiel.

Seit der Mitte des vorigen Jahrhunderts wurden die sozialethischen und geschichtsphilosophischen Bemühungen in steigendem Masse von Ideen der englischen und französischen Philosophie beherrscht. Aber seit etwa zehn Jahren mehren sich allenthalben die Versuche, auch für die Probleme der Sozialphilosophie den deutschen philosophischen Idealismus und besonders das Werk Kants nutzbar zu machen. In dieser Richtung bewegen sich drei ethisch-soziologische Arbeiten Woltmauns, an denen die „Kantstudien" nicht flüchtig. vorübergehen durften. Mlit ungewöhnlicher synthetischer Kraft ist Woltmann . daran gegangen, die Hauptrichtungen des modernen Lebens und Denkens, die Grundgedanken der neueren deutscheu Philosophie, der Entwicklungstheorie und Wirtschaftslehre zu einem ästhetisch-ethischen Monismus zu vereinigen. Auf dem, Grunde des transzendentalen Idealismus errichtete er ein umfangreiches sozialphilosophisches Lehrgebäude.

I.

Ludw. Woltmann, Dr. med. et phil., System des moralischen Bewusstseins mit besonderer Darlegung des Verhältnisses der kritischen Philosophie zu Darwinismus und Sozialismus. Düsseldorf, Herm. Michels Verlag. 1898. VIII u. 397 S.

Jas Buch atmet den Geist der deutschen idealistischen Philosophie und bezengt gleichzeitig eine eindringliche Bekanntschaft mit neueren Ideon und Beobachtungen über das gesellschaftliche (ieschehen. In grossen Zügen entwirft der Verfasser zuerst eine 'Theorie der moralischen Erfahrung, danach eine Entwicklungsgreschichte des moralischen Bewusstseins und schliesslich eine I aurstellung vom Inhalte des sittlichen Lehens. Z/u derselben Zcit, als das vorliegende Werk orschicn, , veröffentlichte ich einen 
Versuch, den m. E. zentralen Begriff der Moraltheorie, den Begriff des unbedingt Wertvollen psychologisch zu vestinmen. Bei dieser Gelegenheit erklärte ich, es sei notwendig, alle bisherige Ethik (einschliesslich der Kantischen) mit den Mitteln der Kantischen Erkenntnistheorie zu revidieren. Vollends eine so unfassende systematisch-monistische Absicht, wie die eingangs angedeutete Woltmanns, setzt auf Schritt und Tritt die Ergebnisse des erkenntnistheoretischen Kritizismus voraus. Woltmann war sich dessen deutlich bewusst. Die erkenntuistheoretische Grundlage seines Systems (Kap. I, In) entstammt der Kantischen Philosoplie; ebenso die Deduktion der formalen ethischen Principien. Der Terfasser steht auf dem Boden des Neukantianismus, nahe bei Riehl und Cohen. Seine ganze Arbeit ist ein schöner Beweis von der unerschöpften Fruchtbarkeit Kantischer Gedanken. - Wenn ich an dem angegebenen Orte des Weiteren eine psychologische Analyse und Kritik der Hauptbegriffe Kants, anch des Erkemntnistheoretikers, forderte (und teilweise in Angriff nahm), so ist Woltmann von dieser Notwendigkeit nicht gleichermassen iiberzengt. Danit ist der wichtigste, vielleicht der einzig prinzipielle Gegensatz zwischen dem Neukantianismus und der empirischen, erkemntnistheoretisch sich besinnenden Psychologie berührt.

Die grundlegende Erörterung Woltmanns über das Verhältnis zwischen kritischer und genetischer Nethode ist für mich unbefriedigend, trotz mancher wertvollen Bemerkungen über das Unterscheidende der beiden, vollends trotz des schliesslichen Hinweises auf Platons halb mystische Lehre von der Wiedererimnerung und deren geistreicher Verknüpfung mit Häckels biogenetischem Grundgesetz. Die kritische Methode fällt hier einfach mit der erkenntnistheoretischen zusammen; die genetische soll zu einer Entwicklungsgeschichte des natürlichen und geistigen Lebens fülıren. Unter den genetischen Wissenschaften vom Leben wird die Psychologie gewöhnlich nicht mit angeführt, gelegentlich aber wird sic dorthin gerechnet. Gehört die Analyse des entwickelten Bewusstseins und die Erkenntnis seiner allgemeingültigen Formen nicht zu den Aufgaben der Psychologie? Der innere Zusammeuhang zwischen philosophiseher Prinzipienlehre und Entwicklungstheorie wird meines Erachtens durch eben diese aualytische Psychologie vermittelt.

Schon der alles beherrschende Begriff des „Bewusstseins“ erfübrt bei Woltmam nirgends die psychologische Bestimumng, 
deren er bedarf. Bald wird darunter (S. 5) die Funktion des Urteilens und Begreifens verstanden, bald die logische Selbstbesinnung, bald das System der allgemeingültigen Gesetze oder (was wiederum nicht geschieden wird) der Formen der Vermunfthätigkcit. Sehr häufig rückt daher die ethische Diskussion unter einen einseitig intellektualistischen Gesichtspunkt. Die Begriffe „Bewusstsein" und "Vernunft" werden gewöhnlich geradezu gleichgesetzt. Die Folge ist trotz, ja z. T. wegen der ungewöhnlichen stilistischen Gewandtheit des Verfassers, eine oft peinliche Vieldeutigkeit seiner Sätze; z. B. bei der fundamentalen Frage nach dem Verhältnis der praktischen zur theoretischen Vernunft. Eine erschöpfende jsychologische Analyse jenes Begriffes scheint mir noch immer zu den dringendsten Aufgaben der Moralwissenschaft zu gehören.

Bestände die Funktion der praktischen „Vernunft" einfach darin, dass wir Ergebnisse der theoretischen Vernunft (Begriffe oder Urteile) auf die elementaren Begehrungen und Triebe anwendeten oder übertrügen, so wäre das Sittliche nicht identisch mit dem Vernünftigen. Die sittliche Vernunft hat ihr eigenes Apriori (d. h. Funktionsgesetz), weil das Willensleben unmittelbar zu eignen Formen sich gestaltet, ohne Vermittlung irgend eines logischen Gesetzesbewusstseins. Die psychologischen 'Thatsachen der Wertbildung kommen bei Woltmann, wie bei den meisten kritizistischen Ethikern zu kurz. - Der Verf. ist mit allen bedeutenden Kantianern der Gegenwart einig in der Ablehnung der theologischen Metaphysik Kants; er erklärt die immanente Deutung, die der Begriff der praktischen Vernunft durch Fichte erfuhr, für einen Fortschritt (S. 54). Aber seine Kritik ist Kanten gegenüber nicht entschieden genug. Das „Ding an sich“ als völlig „beziehungslos" zur sog. Sinnenwelt bleibt unangetastet, und damit auch die zur Hälfte metaphysische Lehre von der transcendentalen Freiheit.

In dem Kapitel (IV) über die Freiheit des Willens stehen von den bisher aufgetretenen Freiheitsbegriffen alle wichtigeren ungeordnet neben einander. Besonders werden die Freiheit in Sinne der Ursachlosigkeit und die sittliche Freiheit ebensowenig geschieden, wie die beiden zugehörigen Begriffe der Notwendigkeit.

Das "System der Zwecke und Gefühle" im folgendeu Kapitel enthält einc rein logische systematik. Was soll man sich bei dem satze denken: „Fs giebt nur bewusste Gefïhle und insofer'n sie? 
bewusst sind, sind sie den logischen Gesetzen des Bewusstseins unterworfen"? (Man vergleiche andrerseits im folgenden, S. 90: „Das Geschmacksurteil über das schöne Objekt ist ein freies Wohlgefallen".) Der Begriff des Wertes spielt gelegentlich hier hinein, ohne irgendwie näher bestimmt zu werden. Das Ganze leidet an einem Übermass von Architektonik, die durch die enge. Anlehnung an Kantische Schemata nicht wertvoller wird. Die sehr summarische Erörterung des Zusammenhanges zwischen Gefühlen und Begriffen gipfelt in dem Satze, „dass es keine Logik ohne Ästhetik giebt". So redet der Verfasser nach der Art erkenntnistheoretischer Formalisten auch sonst zuweilen, besonders, wo es sich um psychologische Fragen handelt, lieber von allgemeinen Begriffen und deren Verhältnissen, als von den Thatsachen, die da begriffen werden sollen. Das erleichtert die Systematik, verflacht aber die Probleme.

Die beiden Kapitel „Ethik (= Sittlichkeit) und Religion“, „Ethik und Kunst" (sollte heissen: Sittlichkeit und Schönheit) verknüpfen in schöner Beredsamkeit die Principien der Kritik der Urteilskraft mit den Ideen der deutschen und englischen Humanitätsphilosophie und mit dem ästhetisch-ethischen Ideal Schillers. Hieran schliesst sich eine Synthese im Sinne Platons, Schillers und Goethes zwischen Schönheit und Religiosität. In diesen klaren und herzlichen, durch Kants Teleologie geläuterten Ausführungen bleibt nur das Verhältnis der Liebe zur Schönheit etwas unbestimmt und schwankend, auch abgesehen von dem beinahe unüberbrückbaren Gegensatz zwischen der christlichen áyá $\pi \eta$ und dem.

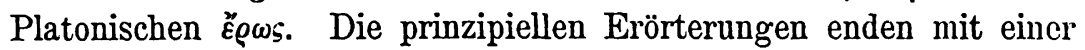
ästhetischen Betrachtung der Philosophie als des „idealen Selbstbewusstseins der Menschheit".

Der Gedanke der genetischen Einheit des Menschengeschlechts und die umfassendere Idee einer stetigen Entwicklung der Kultur aus der organischen Natur beherrschen das zweite Buch. An Herder anknüpfend, aber mit der modernen Descendenztheorie dessen dualistische Schranke überwindend, entwirft der Verfasser eine Vor- und Urgeschichte der Sittlichkeit. Die Darstellung des ${ }_{\text {„Darwinisnuus }}$ " führt thatsächlich über den strengen, der Tendenz nach rein mechanischen Darwinismus hinaus. Der leitende, teleologische Gedanke einer auf Vervollkommnung abzielenden Gestaltungskraft, einer auf sittliche Freiheit gerichteten Zielstrebigkeit des organischen Lebens geht vielmẹr auf Lamarck, Goethe und 
die deutsche Entwicklungsphilosophie zurück. - Nit Herder, Kapp, Noirée und Marx setzt Woltmann die Produktion des Werkzeugs und die Werkzeugthätigkeit in Parallele zur Entwicklung und Funktion der Organe. Aus der Werkzeugthätigkeit leitet er unnittelbar die logischen Funktionen, vor alleu das kausale Denken ab und daraus die Anfänge der Sittlichkeit. Eine genauere Analyse der uns gegebenen psychischen Zusammenhänge hätte hier, wie beinahe überall, zu einer weniger einfachen, aber auch weniger intellektualistischen Auffassung geführt. - Interessant ist in folgenden (IV, 6) die Zusammenstellung von Benierkungen Kants über eine Geschichte der Freiheit und praktischen Vernunft. Diese oft wenig beachtete Richtung des Kantischen Denkens trat allerdings für Kant selbst, namentlich in der kritischen Periode weiter zurück, als die vorliegende Darstellung den Anschein erweckt; aber die hier vereinigten, zerstreuten Aussprüche des Philosophen mögen den oberflächlichen Empirikern und nicht minder den formalistischen Erkenntnistheoretikern zu denken geben, die den kritischen und den entwicklungsgeschichtlichen Standpunkt schlechtweg für Gegensätze halten. Es folgt eine vergleichende Geschichte der moralischen Ideen in Dichtung, Religion und Philosophie. Der Historiker von Fach wird hier gar oft bedenklich den Kopf schütteln und sich an eine überwundene, von der nachkantischenIdeenlehre beherrschte Geschichtsauffassung erinnert finden. Ich hebe nur die Idee vom Reiche Gottes hervor, die in den Mittelpunkt der christlichen Ethik gestellt ist (VIII). Sie wird völlig modern im Sinme einer immanenten und autonomen Moralphilosophie gefasst, ohne Rücksicht auf die historischen Bedingungen des Christentums. „Das Reich Gottes ist ein Gericht, das Verblendung und Thorheit in eine jenseitige Welt verlegt hat." 'Thatsächlich hat Christus, wie das durch seine eigenen Aussprüche bewiesen und auch von der neueren Theologie anerkannt wird, das Reich Gottes und das Weltgericht fast nur oder vielleicht ganz ausschliesslich im jenseitigen, eschatologischen und eudämonistischen Simne verstanden. Aber jede Gregenwart hat ja das Recht, dic Gedanken und Werte der Vorzeit dem eigenen Leben zuliebe umzudeuten. Und Woltmann hebt mit gutem Takte aus den historisch überlieferten Ideenkreisen das hervor, was, soweit wir heute sehen können, sachlich das Wesentlichste ist. - Das Mittelalter als das „Jahrtausend moralischer Finsternis“ wird mit „traurigem schweigen" übergangen. Ebensowenig. kommen der Mystizismus, 
der Pietismus und die Romantik (auch die neueste), kurz alle die fruchtbaren Epochen und Tendenzen einer ausgesprochenen Vorherrschaft des Geinütslebens zum. Worte. In der Darstellung der Reformation fällt nur ein heftiges und unhistorisches Urteil über Luthers Verhalten im Bauernkriege besonders auf. Der deutsche Humanismus und Idealismus werden mit wenigen Strichen skizziert. Ein kurzer Abschnitt über den Sozialismus, nur in der Auffassung der Geistesgeschichte von Marx abweichend, führt zur „Ethik der Gegenwart" über. Nach einigen einleitenden Sätzen über die Notwendigkeit ethischer Selbstbesinuung versucht der Verf. aus den Lehren Tolstois und Nietzsches das Zukunfttragende herauszuheben. Von Nietzsches Moralanschauung wäre gerade in diesem Buche ein gleichmässiger ausgefülnrtes und abgerundeteres Bild willkommen gewesen. Mit lebhafter Zustimmung begrüsste ich die starke Hervorhebung des frühen und meist zu wenig gewürdigten Aufsatzes „Über den Nutzen und Nachteil der Historie für das Leben".

Das letzte Buch, vom Inhalte des sittlichen Lebens, steht unter den Zeichen Darwins und Marxens. Ich übergehe die teilweise höchst beachtenswerten Ausführungen über das T'erhältnis des Kritizismus zu den biologischen und sozialen Problemen der Gegenwart, weil der Verf. diese Fragen in zwei besonderen, nachher zu besprechenden Schriften ausführlicher behandelt hat. - Im II. Kapitel "Das System der Triebe und Bedürfnisse" finden sich Ansätze zu einer willenspsychologischen Analyse der Noralthatsachen, aber leider nur unzureichende Ansätze. Ohne tieferen Zusammenhang stehen der Geschlechtstrieb, ein Trieb zur Selbsterhaltung, zur Vorstellung und ein Bildungstrieb nebeneinander. Bei den drei letzten wäre eine viel feinere psychologische Differenzierung nötig gewesen. Die Arbeit wird zu äusserlich als Werkzeugthätigkeit verstanden. Der Bildungstrieb wird mit Schiller als "Formtrieb" den Stofftrieben gegenüber gestellt. Wertvolle Motive eines moralpsychologischen Begreifens verklingen zu rasch in den vieldentigen Namen Spontaneïtät, Vernunft, Trieb nach Wahrheit, Freiheit, Schönheit. Die Lehre von den Affekten sucht den Einheitspunkt von Glückseligkeit und Sittlichkeit da, wo er ganz gewiss allein gefunden werden kann: in der ästhetischen Kultur und in Genusse des ästhetischen Schraffens. - In der allgemeinen Erörterung des Verhältnisses von Individuum und Gesellschaft finde ich eine vor vier Jahren 
erhobene und theoretisch begründete Forderung auch bei Woltmann: die Ausdrücke Egoismus und Altruismus. seien als unbrauchbar aus der Ethik zu verbannen.

$\mathrm{Zu}$ den einzelnen Gebieten der angewandten Ethik (Wirtschaft, Staat, Recht; Ehe, Familie, Beruf der Frau; Persönlichkeitskultur und Erziehung) können wir dem Verf. hier nicht folgen, wie lohnend es auch wäre. Im ersten Buche hatte er die bekannten drei Formulierungen des kategorischen Imperativs in engem Anschluss an Kant, ohne Kritik nach einander entwickelt. Jetzt, wo es sich um Anwendungen und Konsequenzen des obersten Moralprinzipes handelt, treten die erste und die dritte Formulierung gänzlich zurück. Soweit Woltmanns Ausführungen hier wirklich auf Kants Ethik basieren, gehen sie durchweg auf die zweite, früher an dritter Stelle erörterte Fassung des kategorischen Imperativs zurück, wonach in jeder Person die Menschheit jederzeit als Selbstzweck soll geachtet werden. Ich erblicke hierin eine neue Bestätigung meiner Ansicht, dass diese Fassung des Prinzipes moralischer Beurteilung nicht in demselben Sinne formal ist, wie die beiden andern, und dass sie - was schon die Kritik der Urteilskraft und neuerdings fast jede auf Kant zurückgehende Ethik beweist -, die allein fruchtbare ist.

Üngern und erst nach dem Studium seiner übrigen Schriften entschloss ich mich, gegen Woltmanns Hauptwerk einzelne Bedenken geltend $\mathrm{zu}$ machen. Es besitzt Vorzüge, die nur durch reichliche Citate wiederzugeben wären. Dahin gehört die sichere Bezeichnung der wesentlichen Fragen und Zusammenhänge, der knappe, lebendige und oft überraschend einfache Ausdruck der Ergebnisse, besonders auch die neue Fassung alter, bisher weniger gut gesagter Wahrheiten. Lässt man das Ganze in seiner stilvollen Geschlossenheit auf sich wirken und bedenkt den Umfang der gestellten Aufgaben, so erscheint das Buch als die wertvollste philosophische Leistung des theoretischen Sozialismus und als eine der bedeutsamsten Erscheinungen der neueren Ethik. 
II.

Ludw. Woltmann, Dr. med. et phil., Die Darwinsche Theorie und der Sozialismus. Ein Beitrag zur Naturgeschichte der menschlichen Gesellschaft. Düsseldorf 1899. Herm. Michels Verlag. IV u. $397 \mathrm{~S}$.

Bekanntlich hat der Darwinismus seit nahezu 30 Jahren gerade unter den deutschen Sozialisten eine rasch zunehmende Verbreitung gefunden. Das hatte mannigfaltige Gründe, z. T. recht ïusserliche, wie die Gemeinsamkeit des Gegensatzes gegen die kirchliche Orthodoxie, z. T. viel tiefer liegende. Es ist ebenso bekannt, dass zahlreiche Darwinisten, darunter tüchtige Zoologen, die Lehre von der natürlichen Auslese im Kampf ums Dasein benutzt haben, um die Forderungen des Sozialismus einzuschränken oder in Bausch und Bogen abzulehnen. Auch Nietzsche, der in weitem Umfange und ziemlich kritiklos Darwinistische Gedanken auf das Kulturproblem übertrug, war ja gleichzeitig dem politischen Sozialisnus abgeneigt. Nietzsches eigene Lehren werden von sozialen Revolutionären wie von unbedingten Verteidigern des gegenwärtigen Wirtschaftssystems sowohl verlästert, als gelobt. Und dieses schliessliche Erhobensein über die wirtschaftlichen Kämpfe des Tages spricht für die übergreifende Bedeutung der Nietzscheschen Gedanken. Darwin hat nur mit äusserster Zurückhaltung solche Fragen berührt, die jenseits der biologischen Wissenschaft (im engeren Sinne des Wortes) lagen. Obwohl von der Bevölkerungslehre des Malthus her ihm Anregungen kamen, gestand er, mit den Thatsachen und Zusammenhängen des wirtschaftlichen Lebens wenig vertraut $\mathrm{zu}$ sein. Die ethischen Konsequenzen,. die er selbst seiner Entwicklungstheorie gab, erschöpften sich in der bekannten hypothetischen Ableitung des sympathischen Gefühls, und seime eigene Moralanschauung gipfelte in in dem Satze, womit noch heute mancher den ganzen Sinn der Kantischen Ethik zu umfassen meint: „Was ihr wollt, dass man euch thue, das thut auch anderen". Haeckel und der scharfsinnige sozialist Kautsky begegnen sich in dem Urteil, dass der Sozialismus mit dem Darwinismus nichts $\mathrm{zu}$ thun habe. Versteht man unter Darwinismus die konkret vorliegende Theorie Darwins, so muss man diesem Urteil beistimmen.

Aber der Fntwicklungsgedanke hat anch eine philosophische, genauer: eine psychologische und ethische Bedeutung. Dieser Ge- 
danke ist ja aus der Geistesphilosophie erst auf das Naturgeschehen übertragen worden; jetzt wendet er sich mit bereichertem Inhalte auf die Probleme des Kulturlebens zuriick. Auf der anderen Seite liegen der Wirtschaftslehre und Geschichtswissenschaft mehr noch als der Biologie psychologische Voraussetzungen zu Grunde, und alle sozialen Fragen sind zugleich moralwissenschaftliche. - Woltmann setzte sich die dreifache Aufgabe: „1) eine litterar-historische Übersicnt über die Problemstellung zu geben, wie bisher das Verhältnis des Darwinismus zum Sozialismus aufgefasst worden ist; 2) die allgemeinen naturgeschichtlichen Grundlagen der Sozialund Geschichtswissenschaft $z u$ entwickeln, und im Anschluss daran 3) das spezielle Problem zu behandeln, ob die Darwinsche 'l'heorie von der natürlichen Zuchtwahl im Kampf ums Dasein mit den historischen und wirtschaftlichen Lehren des Sozialismus harmoniere oder nicht." Das Buch wird von der dritten Frage beherrscht, und von der Überzeugung, dass sie positiv zu beantworten sei. Zuweilen steht dem Philosophen und namentlich dem Historiker Woltmann der Parteimann im Lichte. Die ganze Arbeit ist weniger ausgereift und schon schriftstellerisch unfertiger als die vorhergegangene.

Von der umfänglichen Litteratur ist die deutsche nahezu vollständig, die englische zum besseren Teile verarbeitet. Die Kulturfragen, um die es sich hier handelt, sind ja mit den Mitteln der Naturwissenschaft allein keineswegs zu lösen; Treitschke warnte zuweilen vor den politisierenden und ethisierenden Naturforschern. Die sozialistischen Anhänger Darwins sind ihren Darwinistischen Gegnern im Allgemeinen an ökonomischer und philosophischer Bildung überlegen. Bei diesen wiederholen sich immer dieselben Unklarheiten und Verwechslungen: sie identifizieren den Kampf um die wirtschaftliche Existenz einfach mit dem tierischen Kampfe ums Dasein und den Begriff des am besten Angepassten mit dem des wirtschaftlich Tüchtigsten oder gar des moralisch Besten; ferner; wozu freilich sozialistische Schriftsteller noch gegenwärtig vielfach Anlass geben, verwechseln sie die Sozialisierung der Produktionsmittel mit der Abschaffung alles Privateigentums und deuten die Forderung gleicher politischer Rechte und kultureller Entwicklungsbedingungen dahin, dass der Sozialismus die qualitative Gleichheit aller Menschen behaupte oder erstrebe. Diese Missverständnisse sind schon oft, auch von sehr bedingten Anhängern, ja von Gegnern des ökonomischen Sozialismus klargestellt 
worden, so von Lange, Schäffle, Wagner, Schmoller, Büchner, Ritchie; Wallace, Haycraft, Ploetz, Huxley. Im Anschluss an diese und andere Antoren bekämpft Woltmann die angedeuteten Irrtüner und ihre Konsequenzen, mit beinahe ermüdender Eindringlichkeit, oft leidenschaftlich wie ein Agitator, immer vlar und gewandt.

Die systematische Erörterung des Verhältnisses zwischen naturgeschichtlicher und historisch ökonomischer Entwicklung begiunt mit einer knappen und kundigen Darlegung der Prinzipien der neueren Descendenztheorie (5. Abschn.). In der Vererbungsfrage wird eine Vermittelung zwischen Weismann und Spencer angestrebt. Der von Spencer, Schäffle, Lilienfeld u. a. entwickelten „organischen“ Auffassung der Soziologie schliesst der Verf. sich kritisch an, in Sinne einer induktiven und genetischen Analogie zwischen Organismus und Gesellschaft. „Der soziale Organismus ist eine Fortbildung des individualen Organismus zu einer höheren Stufe und Verbindung des organischen Lebens. Freilich konmt es darauf an, die Vermittelungen und Zwischenglieder festzustellen, durch welche diese Höherbildung ermöglicht wird“. Diese Mittelglieder seien technische und psychische Faktoren. Zur ergänzenden Weiterbildung der Marxistischen Geschichtsphilosophie, (die in seinem dritten, schliesslich zu betrachtenden Werke ausführlich behandelt wird) fordert Woltmann eine „tiefere Erforschung des Zusammenhanges der Physiologie einerseits mit der Technologie und Psychologie andrerseits". In dieser Hinsicht kommt seine eigene Gesellschaftslehre (Abschn. 6) freilich über die physiologischen und logischen Analogien der Kapp, Geiger, Noirée nicht wesentlich hinaus; die psychologische Analyse der technischen Thatsachen und des wirtschaftlichen Lebens überhaupt bleibt unzureichend.

Im letzten Abschnitt (7) zieht der Verf. aus dem Vorangegangenen politische und soziale Konsequenzen. Bei der Frage der Rassenauslese kommt er auf Kolonisation und Krieg zu sprechen und widerlegt mit Darwins eigenen Worten die Darwinistischen Phrasen der Gewaltethiker; nur machen sich fertige moralische Werturteile hier zu breit an Stelle des sachlichen histórisch-soziologischen Begreifens. Weiterhin werden noch einmal im Zusammenhange die wichtigen Merkmale hervorgehoben, wodurch die sog. freie Konkurrenz des privatkapitalistischen Wirtschaftssystems sich vom zatürlichen Kampf ums Dasein unterscheidet. Schliesslich 
stellt Woltmann die Hauptthatsachen des Wirtschaftslebens (Eigentum, Freiheit, Wettbewerb), die Bevölkerungss und Frauenfrage, das Problem der sozialen Differenzierung unter den Gesichtspunkt des sozialistischen Ideals und eines erkenntnistheoretisch geklärten Darwinismus.

Gleich im Eingange seines Buches fordert der Verf., dass der theoretische Sozialismus sich auf Kant besinne. Im weiteren beruft er sich nur selten ausdrücklich auf Kantische Lehren, (auf das Apriori des reinen Verstandes und auf die Ideen zu einer Entwicklungsgeschichte der Vernunft). Aber sein ganzes Unternehmen ist geleitet von der fruchtbaren Überzeugung, die jeder rechte Schüler Kants teilen muss: dass die notwendige theoretische Verknüpfung der naturgeschichtlichen mit der geistig-sozialen Entwicklung auf dem Boden der kritischen Philosophie und nur auf diesem Boden möglich ist.

\section{III.}

Ludw. Woltmann, Dr. med. et phil., Der historische Materialismus. Darstellung und Kritik der Marxistischen Weltanschauung. Düsseldorf 1900. Herm. Michels Verlag. VI u. $430 \mathrm{~S}$.

Seitdem Bernstein neuerdings die theoretischen yoraussetzungen der Sozialdemokratie einer umfassenden Kritik unterzogen und seine Parteigenossen auf die Kantische Philosophie hingewiesen hat, ist in deren Reihen der Streit um Kant nicht mehr zur Ruhe gekommen. Was vor 30 Jahren von F. A. Lange angebahnt, was später von Cohen, Natorp, Stammler, Staudinger, Vorländer und anderen Kantianern auf verschiedenen Wegen erstrebt wurde: eine Durchdringung des Sozialismus mit den Prinzipien der kritischen Philosophie, das scheint jetzt in den führenden Geistern der sozialdemokratischen Partei zur Wirklichkeit werden zu wollen. - Auch Woltmanns jüngstes Buch steht, wie das nach dem Entwicklungsgange des Verfassers zu erwarten war, „unter dem Zeichen der Rückkehr zu Kant". Es will dem dringlichen Bedürfnis nach einer historischen und kritischen Darstellung des Marxismus dienen.

Von dessen philosophiegeschichtlichen Voraussetzungen (1.Teil) wird die Kantische Philosophie am ausführlichsten behandelt. Die fassliche Wiedergabe des Hauptinhaltes der drei Kritiken 
ist allen denen zu empfehlen, die nicht in der Lage sind, die Urtexte im Zusammenhange zu studieren. Fichtes Wissenschaftslehre wird mit liebevollem Verständnis, Schellings Philosophie summarisch und wenig übersichtlich dargestellt. Hegel und Feuerbach haben in Wirklichkeit den neueren theoretischen Sozialismus viel stärker bestimmt, als Kant, den Marx wie Engels nur ungenau kannten und fast durchweg missverstanden. Woltmann ist über die historischen Zusammenhänge, namentlich auf der deutschen Linie, recht genau orientiert. Trotzdem giebt er von Hegels idealistischem Systeme und Feuerbachs Anthropologie verhältnismässig wenig ausgeführte Skizzen und übergeht mit Stillschweigen die philosophischen Quellen des Marxismus, die in Frankreich und England entsprangen. Darin kommt sein kritischer Standpunkt bereits zum Ausdruck.

Mit Recht betont der Verf. immer von Neuem zwei historische Thatsachen, deren Vernachlässigung auch in sozialistischen Kreisen viel Verwirrung angerichtet hat: dass die Marxistische Doktrin in ihrer fünfzigjährigen Entwickelung mancherlei Wandlungen durchgemacht hat; und dass sie zunächst einen stark polemischen Charakter trug, den Charakter des bewussten Gegensatzes einmal gegen die herrschenden Wirtschaftstheorien, zum anderen gegen den Spiritualismus der Hegelschen Philosophie. Es war daher ein philosophisches und litterarhistorisches Verdienst, die Entwicklungsgeschichte des Marxismus von seinen Anfängen bis zur Gegenwart eingehend zu verfolgen. Die praktischrevolutionären Tendenzen der Theorie, ihre von augenblicklichen politischen Bedürfnissen stets mitbedingte und naturgemäss widerspruchsvolle Gestaltung mussten ihrer philosophischen Einheitlichkeit und dem wissenschaftlichen Werte des von ihr einzeln Überlieferten Eintrag thun. Man kann zweifeln, ob von einer Narxistischen „Weltanschauung“ zu reden überhaupt berechtigt ist. Die notwendige Differenzierung der geistigen Produktion setzt auch einem hochbegabten Feuerkopfe Schranken. Und so wird die kritische Geschichte vieles wieder streichen von den Werturteilen Woltmanns, der in Marx einen der grössten Philosophen und Historiker erblickt. Sie wird freilich auch der Ungerechtigkeit gedenken, nit der die Geschichtsschreiber der Philosophie den weitblickenden und scharfsinnigen Mann bisher zu ignorieren pflegten. Wenn indessen der Verf. Marxen ganz nahe an Kant heranrückt, denselben Marx, dem er eine „Degradation des Geiștes“ und die schlimmsten er- 
kenntnistheoretischen Unzulänglichkeiten mit Recht vorwirft, wenn er die wissenschaftlichen Methoden beider als wesentlich identisch anffasst, so wird der Kenner Kants ebenso wie der Historiker der Geschichtstheorie entschicden widersprechen. Woltmann unterstreicht schr stark cinige Zeugnisse dafür, dass Marx (wie alle bahnbrechenden Sozialpolitiker und alle tiefer angelegten Historiker) bei seiner wissenschaftlichen Thätigkeit teilweise von moralischen Wertungen und unbedingten ethischen Urteilen bestimmt war. Aber diese biographisch und psychologisch bedeutsame Thatsache ändert nichts daran, dass alle massgebenden Schriften des Marixismus in ethischer wie in erkenntnistheoretischer Beziehung einen oberflächlichen Relativismus und Sensualismus lehren. Marxens Hauptverdienst um die Kulturgeschichte liegt in der endgiiltigen Eroberung eines vorher arg vernachlässigten Stoffgebietes, in der grundsätzlichen Berücksichtigung und z. T. sehr glücklichen Verknüpfung der wirtschaftlichen Thatsachen, während seine $\mathrm{Me}$ thode über eine kritiklose Nachahmung der Hegelschen Dialektik nicht hinauskam. Der Bannkreis dieser Methode und der ursprünglichen, in der Partei noch heute herrschenden materialistischen Vorurteile wird allerdings gelegentlich durchbrochen durch spätere Modifikationen der Theorie, namentlich in vier nachgelassenen Briefen von Engels, die Woltmann gut that, an dieser Stelle zu veröffentlichen. Hier wird eine Rückwirkung des geistigen „Oberbaues" der Kultur auf den ökonomischen Unterbau ausdrücklich zugegeben; es wird die reale Bedeutung der geistigen Tradition und der kulturelle Wert der sozialen Arbeitsteilung angedeutet; es wird die zunehmende Differenzierung und Verflechtung der idealen wie der materiellen „Ursachen“ hervorgehoben.

Der letzte, umfangreichste Teil des vorliegenden Werkes, die ${ }_{n}$ systematische Kritik des Marxismus" bezeugt eine weitgehende Unabhängigkeit des Verf. von den Parteidogmen. Verdienstlich ist der Hinweis auf die zahlreichen, ganz verschiedenen Bedeutungen des Wortes "historischer Materialismus", die in der Litteratur bunt durch einander gehen. Nur werden sie auch von Woltmann nicht scharf genug aus einander gehalten und nicht, ausreichend definiert. Zuweilen dehnt er den Begriff der materialistischen Geschichtsauffassung so weit aus, dass damit jedes empirische historische Begreifen in Gegensatz zu spekulativen Konstruktionen bezeichnet wird. 'Thatsächlich darf dem historischen ivaterialismus nur in dieser weiten Fassung "unbedingte“ metho- 
dische Berechtigung zugesprochen werden. Woltmanns kritische Einwendungen richten sich fast ausschliesslich gegen Marxens materialistische Auffassung der rein geistigen Entwicklungen. Innerhalb des ökonomischen -Gebietes soll der ökonomische Materialismus unbedingt zu recht bestehen, der alles auf die Produktion und Reproduktion des physischen Lebens (Ernährung, Kleidung, Wohnung, Fortpflanzung) zurückführt. Aber der religiöse, wissenschaftlich denkende, musizierende Mensch ist kein anderer als der wirtschaftende. So wird schon das wirtschaftliche Thun von Motivèn dès persönlichen Ehrgeizes und der traditionellen Pietät, von ästhetischen und ethischen Bedürfnissen mitbestimmt. Woltmann betont in abstrakt räsonnierender Weise, womit er gewiss keinen Marxisten überzeugen wird, die historische Realität und Wirksamkeit einer bewussten allgemeinen Menschheitsmoral. Fruchtbarer wäre es gewesen, die einfache Wahrheit auszusprechen, dass auch die sog. materiellen Bedürfnisse seelische Thatsachen sind, so gut wie die geistigsten; dass ferner alles psychische Geschehen in der natürlichen Einheit des Bewusstseins zusammenwirkt und auf immer neue, höhere, haltbarere Einheiten hinzielt. Der schwache Punkt des Marxismus wird zuweilen in jenem Mangel an Psychologie richtig erkannt, woran schon Marxens philosophischer Stammvater Hegel leidet. Aber die Psychologie ist auch Woltmanns Stärke nicht. Im Mittelpunkte einer Kritik des Marxismus muss das psychologische und historische Verhältnis zwischen dem sog. (ökonomischen) Unterbau und dem Oberbau der Kultur stehen. Das Wort Wechselwirkung sagt hier wie gewöhnlich $\mathrm{zu}$ wenig und $\mathrm{zu}$ viel. Der Verf. lässt wiederum aus dem technischen Verhalten das logische Bewusstsein und daraus alles Weitere hervorwachsen. Die Darstellung bleibt mit ihrem unpsychologischen Schematismus beschränkt auf eine vielfältige Bezeichnung des Problems von der kulturgeschichtlichen Bedeutung der Technik, eines Problemes, das als solches auch Marx deutlich gesehen hat.

Eine erschöpfende Kritik der Marxistischen Geschichtsauffassung würde ein sachkundiges Eingehen auf Zusammenhänge des wirtschaft,lichen Lebens nötig machen. Marx und Engels haben den historischen Mcierialismus oft so gewendet, dass alle Geschichte eine Geschichte der Klassenkämpfe sej. Nach Woltmann ist die Geschichte ebensosehr ein fortgesetzter Kampf gégen die Klassen. Der Verf. entfernt sich hier, wie auch sonst viel- 
fach, weiter vom ökonomischen Materialismus, als er selbst meint. Aber 'an diesem Punkte ist Marx, der Wirtschaftshistoriker, seinem philosophischen Kritiker überlegen. Sicherlich zeigt die Geschichte eine allnälige Überwindung der Klassenunterschiede, eine Entwicklung zur rechtlichen Gleichheit und persönlichen Freiheit. Indessen, mit dem Hinweis auf das Bewusstsein der allgemeinen Menschheitsmoral ist da, wie die Geschichte der Sklaverei und der Leibeigenschaft beweist, theoretisch nichts gethan. Die von Marx beeinflusste Geschichtsbetrachtung betont mit Recht die befreiende Wirkung des sich entwickelnden Privateigentums und der Geldwirtschaft, sowie den entscheidenden Zusammenhang zwischen rechtlicher Freiheit und wirtschaftlicher Intensität der Arbeit. Von alledem ist bei Woltmann keine Rede. Statt den Marxismus durch eine Psychologie des Eigentums, der Arbeit und Produktivität zu ergänzen, verweist er immer wieder auf rein „moralische Ursachen“, auf allgemeine ethische Ideen und auf den schillernden Kantischen Begriff der Spontaneïtät. Kants Freiheitslehre steht unvermittelt neben wertvollen Ansätzen zu einem psychologischen und historischen Begreifen der Willensentwickelung. So wird auch die psychologisch unhaltbare Kategorienlehre ohne jede Kritik dargestellt. Der Zusammenbruchs-, und Verelendungstheorie des Parteisozialismus hält Woltmann nur einzelne Bedenken entgegen, die schon von Marx und Engels ausgesprochen sind. Vor allem hätte aber der hier vorausgesetzte, neuerdings viel umstrittene Begriff des Endzwecks einer erkenntnispsychologischen Kritik bedurft, der die Kantische Lösung der ersten und zweiten Antinomie und sein Begriff der regulativen Idee zugrunde $\mathrm{zu}$ legen war.

Der Hauptwert des inhaltreichen Buches scheint mir in der historischen Darstellung des Marxismus und seiner philosophischen Wurzeln zu liegen. Eine systematische, positive Kritik der Marxschen Lehren, wäre gleichbedeutend mit einer ökonomischen Prinzipienlehre, ja mit einer neuen, auf Kants Erkenntnistheorie gegründeten Geschichtsphilosophie. Sie würde eine einheitliche Entwicklungsgeschichte aller Kulturbedingungen sein und eine psychologische Analyse der Wertbildung voraussetzen. Zur Lösung dieser grossen wissenschaftlichen Aufgabe hat Woltmann, gestützt auf seine grïndliche Kenntnis der kritischen Philosophie, fruchtbare Anregungen gegeben. 